\title{
Hemispheric Patterns in Filament Chirality and Sigmoid Shape over the Solar Cycle
}

\author{
Petrus C. Martens ${ }^{1}$, Anthony R. Yeates ${ }^{2}$, and Karthik G. Pillai ${ }^{3}$ \\ ${ }^{1}$ Physics Department, Montana State University, \\ Bozeman, MT 59717, USA, \\ email: martens@physics.montana.edu \\ ${ }^{2}$ Department of Mathematical Sciences, Durham University, \\ Science Laboratories, South Road, Durham DH1 3LE, UK, \\ email:anthony . yeates@durham.ac.uk \\ ${ }^{3}$ Computer Science Department, Montana State University, \\ Bozeman, MT 59717, USA, \\ email: karthikgp@gmail.com
}

\begin{abstract}
The motivation for our research was to study the correlation between the chirality of filaments and the handedness (S- or Z-shape) of sigmoids. It was assumed that sigmoids would mostly coincide with filaments and that the S-shaped sigmoids would correlate well with filaments of sinistral chirality, which we found that to be at best a very weak relation. Since we had a full solar cycle of filament metadata at hand it was easy to verify the supposedly known hemispheric preference of filament chirality. We discovered that the hemispheric chirality rule was confirmed for the epoch where a thorough manual study had been performed, but that at other phases of the solar cycle the rule seems to disappear and sometimes even reverse.
\end{abstract}

Keywords. Solar Filaments, Solar Sigmoids, Hemispheric Chirality Rule

\section{Introduction}

The overwhelming amount of data coming from the Solar Dynamics Observatory $(\mathrm{SDO}, \approx 1.5 \mathrm{~TB} /$ day), and soon from the Advanced Technology Solar Telescope (ATST) forces the solar physics community to handle observations in a different way from what we are used to. One method of dealing with the data deluge is to develop automated feature recognition codes that produce metadata on solar events and phenomena, catalogs in fact, that until now have been painstakingly put together manually by solar scientists, often graduate students. NASA had foreseen this and funded two teams to develop automated feature recognition codes from prior to the SDO launch in February 2010 .

One of these teams is headed by the first author of this paper and has produced 16 modules for the detection and analysis of different solar features (Martens et al. 2012), see also the more up-to-date websitet. The analysis of the metadata from two of these modules, the "Advanced Automated Filament Detection and Characterization Code (AAFDCC)", see Bernasconi, Rust \& Hakim (2005), and the "Sigmoid Sniffer" (Martens et al. 2012) will be presented in this paper, and the results contain surprises.

Apart from the scientific conclusions, one important lesson we have learned from using the metadata from automated feature detection modules is that it enables us solar physicists to analyze very large datasets in a much more efficient and economical way than we did before. Hence we have the capability now to move away from the analysis of single or

$\dagger$ solar.physics.montana.edu/sol_phys/fft/ 
small sets of events to large and complete sets of data for a given phenomenon. This may lead to the detection of hitherto unknown correlations, and thereby raise now challenging questions and the opportunity to develop new physical insights. The scientific conclusions from this paper are a case in point. The repository of metadata for Solar Physics is the Heliophysics Events Knowledgebase (HEK $\ddagger$ ), and the metadata are accessible from there as well as from the Virtual Solar Observatory 1 .

\section{Filament Chirality}

Sarah Martin and co-workers introduced the concept of chirality for filaments in a remarkable series of papers reviewed by Martin (1998). A key result of their work is that the chirality of filaments - dextral or sinistral - can be determined from $\mathrm{H} \alpha$ observations alone, no magnetic observations are needed. The bearing of the barbs - little sideways protrusions emanating from filaments - can be either left or right (imagine the on- or off-ramps from highways). The dominant direction of the bearing of the barbs determines the chirality of the filament.

As Martin shows filament chirality in essence is the sense of winding of the magnetic field around the axis of a filament (left-handed or right-handed), and hence is related to the force-fee $\alpha$ and the sign of the helicity of filaments.

Bernasconi's AAFDC code detects the barbs of filaments and their bearing, and hence can assign chirality to a filament. The usual attribution criterion is that two more barbs have to be of one bearing versus the opposite one to assign filament chirality. Georgoulis' "Sigmoid Sniffer" (see Martens et al. 2012) likewise detects whether the contour of a sigmoid has an "S" (forward) or a "Z"-shape (reverse).

\section{Filament-Sigmoid Correlation}

We used AIA $94 \AA$ A data from October 112010 until March 27 2011, analyzed by the "Sigmoid Sniffer", and H $\alpha$ data from the same period obtained by Big Bear Solar Observatory, at a rate of about four per day, analyzed by the AAFDC. The correlation matrices, shown in Fig. 1 show that only a small fraction of filaments have an accompanying sigmoid, no surprise since filaments are much more prevalent than sigmoids. More of a surprise is that $2 / 3$ of sigmoids do not have an accompanying filament. Perhaps the reason is that cool filament material has evaporated by the time a sigmoid forms, although there are clear examples of the opposite (e.g. Fig. 2 in Martens \& Zwaan, 2001). The low cadence of the $\mathrm{H} \alpha$ observations may have something to do with that as well; future versions of AAFDC will analyze images from ground stations worldwide.

The correlation between dextral filament chirality and inverse S-shape for sigmoids (and v.v.) seems clear, but the accuracy of prediction is only $64.4 \%$, while the $\phi$-coefficient (see Cramer, 1946, p. 282, second paragraph) of the matrix is only -0.25 indicating a statistically very weak to non-existent negative correlation.

\section{Hemispheric Rule for Chirality}

Fully aware of the hemispheric preferences for filament chirality published by Pevtsov, Balasubramaniam \& Rogers (2003) for the period July 15, 2000 to June 22, 2002, and closely reproduced for the same dataset by the AAFDC (Bernasconi et al. 2005) we used AAFDC metadata to verify the hemispheric rule for the dataset described above. To our

$\ddagger$ http://www.lmsal.com/hek/

I http://sdac.virtualsolar.org 


\section{Experimental Results}

\begin{tabular}{|c|c|c|}
\hline \multicolumn{2}{|l|}{ Colocation Rule } & Conditional Probability \\
\hline Sigmoid $\Rightarrow$ Filament & & $35 \%$ \\
\hline Filament $\Rightarrow$ Sigmoid & & $8 \%$ \\
\hline Shape-Chirality & Dextral & Sinistral \\
\hline Forward & $16.75 \%$ & $41.36 \%$ \\
\hline Inverse & $23.04 \%$ & $18.85 \%$ \\
\hline
\end{tabular}

Figure 1. Correlation between filaments and sigmoids.



Figure 2. Linear fit of percentage of chirality in 15 degree latitude bins.

great surprise we discovered that there was absolutely no dependence of filament chirality on latitude for the period October 112010 to March 27 2011. In Fig. 2 our counts are presented in 15 degree latitude bins, and the result speaks for itself. A large percentage of filaments has undetermined chirality which is normal for solar minimum when filaments typically carry only few barbs. We found the same latitude independent distribution for sigmoid shape, not shown here, with no undetermined values in that sample.

This result motivated us to study the hemispheric chirality preference for all the AAFFDC data available for solar cycle 23 and the beginning of cycle 24, the period from the beginning of 2001 to the spring of 2012. The results are shown in Fig. 3. Our concern about the validity of the result in Fig. 2 was alleviated by that result because the analysis of the AAFDC metadata again reproduced the results from the study by Pevtsov et al., as well as the earlier results from the AAFDC. Clearly there is a variation of the hemispheric rule with the phase of the solar cycle 23. During the extended solar minimum AAFDC had a hard time detecting the barb bearing of the limited number of filaments at all. For several months (around the 2007 tick mark in Fig. 3) during the two years prior to the minimum, 2006 and 2007, it seems that the hemispheric preference was reversed, a very puzzling result, and at the onset of cycle 24 the hemispheric pattern had difficulty reestablishing itself, a considerable amount of negative chirality remains in the northern hemisphere. It is important to keep in mind here that the hemispheric chirality preference does not reverse from cycle to cycle, as was established by Martin, Billimoria \& Tracadas (1994). 


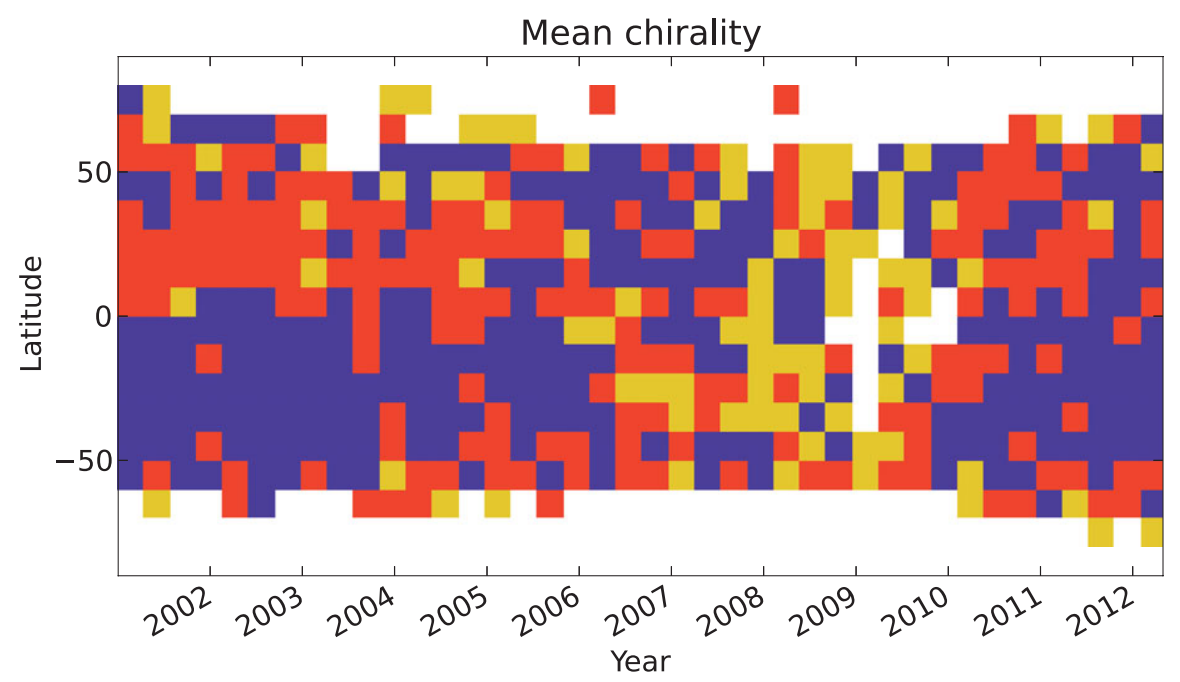

Figure 3. Mean chirality per time-latitude box for 2001-2012. Red (black) indicates dextral chirality, blue (dark grey) sinistral, and yellow (light grey) denotes undetermined chirality.

\section{Conclusions}

We have demonstrated, from a limited three month data set, that there is a weak correlation between the shape of sigmoids ("S" or "Z") and the chirality of the corresponding filaments. We also found from the same dataset that for $2 / 3$ of the detected sigmoids no corresponding filament was detected, and that there was no hemispheric preference law for filament chirality and sigmoid shape in the period of observation.

The most surprising and intriguing result of our analysis is that in the epoch from early 2001 to early 2012 the hemispheric chirality preference law for filaments waxes and wanes; sometimes it is strongly present, at other times wholly absent, and sometimes even reversed. These results are preliminary; we intend to verify the AAFDC produced metadata with manual studies for appropriate time intervals, and we will carefully consider the statistical underpinnings of our results.

\section{References}

Bernasconi, P. N., Rust, D. M., \& Hakim, D. 2005, Sol. Phys., 228, 97

Cramer, H. 1946, Mathematical Methods of Statistics (Princeton: Princeton University Press,)

Martens, P. C. \& Zwaan, C. 2001, ApJ, 558, 872

Martens, P. C. H., Attrill, G. D. R., Davey, A. R., Engell, A., Farid, S., Grigis, P. C., Kasper, J., Korreck, K., Saar, S. H., Savcheva, A., Su, Y., Testa, P., Wills-Davey, M., Bernasconi, P. N., Raouafi, N.-E., Delouille, V. A., Hochedez, J. F., Cirtain, J. W., Deforest, C. E., Angryk, R. A., de Moortel, I., Wiegelmann, T., Georgoulis, M. K., McAteer, R. T. J., \& Timmons, R. P. 2012, Sol. Phys., 275, 79

Martin, S. F. 1998, Sol. Phys., 182, 107

Martin, S. F., Billimoria, R., \& Tracadas, P. W. 1994, in Solar Surface Magnetism, 303-338

Pevtsov, A. A., Balasubramaniam, K. S., \& Rogers, J. W. 2003, ApJ, 595, 500 